\title{
Estudio observacional de habilidades quirúrgicas en residentes
}

\section{Observational study of surgical skills in residents.}

\author{
Eduardo B. Arribalzaga y Patricio F. Jacovella
}

Departamento de Cirugía, Hospital de Clínicas, Facultad de Medicina, Universidad de Buenos Aires, Argentina

Introducción: existen actualmente "agujeros negros" en la transmisión de conocimientos aplicables que obligan a poner atención en los mecanismos de adquisición de destrezas y habilidades. El objetivo es evaluar características cualitativas de las habilidades en 2 tipos de procedimientos técnicos básicos al alcance de un cirujano general.

Material y Métodos: estudio preliminar exploratorio observacional prospectivo en un Hospital Universitario consistente en la observación de los pasos de una toracotomía axilar o una dermolipectomía abdominal anterior con neo ombligo. Se analizó la práctica de las operaciones programadas, no discriminando que médicos residentes las habían efectuado. Los datos se medían en una lista de cotejos con una escala tipo Likert modificada de 3 items. Cada paso de la técnica observada era evaluado sin considerar el tiempo de duración de cada maniobra. Los evaluadores eran ajenos al equipo quirúrgico actuante ubicados donde no se los veía (en miradores) para evitar sesgos o maniobras que afectaran la actividad. Se usaron pruebas estadísticas de ANOVA y prueba de $t$ para detectar validez y diferencias en los resultados.
Correspondencia:

Eduardo B. Arribalzaga

Avda Rivadavia 4282 Piso $4^{\circ}$ Depto $\mathrm{H}$

CP: C1205AAP Buenos Aires Argentina

e-mail: piedralta3@hotmail.com
Resultados: observada una toracotomía, todos los pasos quirúrgicos fueron evaluados por encima del valor 2 (bueno), sin variaciones en el desarrollo de cada paso ni existir diferencias significativas en la comparación de destrezas. Similares resultados se hallaron en la observación de la otra técnica. Conclusiones: con un sencillo método de observación directa inadvertida por el evaluado con criterios específicos se hizo un diagnóstico de situación demostrando la existencia de habilidades adquiridas en cirujanos en formación durante programas de residencia.

Palabras clave: cirugía- enseñanza- residencia -habilidadesevaluación

Introduction: Some " black holes" in the knowledge transmission process, suggest to pay attention on the correct way to acquire specific surgical skills. The objective is to evaluate qualitative features of main surgical skills in two basis surgical techniques performed by residents.

Material and method: Observational prospective preliminary study in University Hospital, in both thoracotomy and abdominoplasty performed. Through a modified comparison Likert scale, specific maneuvers were evaluated. Observers did not know surgeons identity. Statistical analysis (ANOVA and Student $t$ test) was used to detect results validity and differences. Results: Both surgical techniques obtainded a number 2 value (good), without significant differences between them. 


\section{Conclusions: A simple observational method allowed a situational diagnosis and demonstrated the importance of developing teaching skills in surgical residence program.}

Key words: surgery, surgical skills, residents training, evaluation

\section{INTRODUCCIÓN}

En el actual sistema de atención sanitaria existen "agujeros negros" en la transmisión de conocimientos aplicables ${ }^{1}$. Recientes casos de gran incompetencia quirúrgica se vieron en Inglaterra ${ }^{2}$, que dañaron la confianza del público. La sociedad demanda que la buena práctica médica requiera de instrumentos que extiendan la limitada capacidad de la inteligencia humana, entendida ésta como la capacidad de resolver problemas ${ }^{3}$ y evitar errores potencialmente prevenibles ${ }^{4}$.

Los esfuerzos en la medición y predicción del desempeño clínico de un médico joven hicieron foco en indicadores de conocimientos o relaciones entre predictores basados en aprendizaje académico y variados criterios de "performance" profesional ${ }^{5,6}$. Otros estudios incluían mediciones no cognitivas como las actitudes, personalidad y/o experiencia ${ }^{7}$.

Así como puede existir una energía permanente e individual del graduado en medicina por mantenerse competente a lo largo de su vida profesional ${ }^{8}$, hay que poner la atención en los mecanismos de adquisición de destrezas y habilidades a lograr en los primeros años de graduado, más allá de aquellas que son innatas ${ }^{9}$.

Los métodos para enseñar y evaluar las destrezas técnicas a cirujanos en formación persisten vagos, ambiguos y poco fomentados o desarrollados ${ }^{10}$. La cirugía, como ciencia y arte, continúa con un progresivo y avasallante avance desde lo científico y técnico; por consiguiente es necesario comprender las nuevas habilidades a obtener desde lo visualespacial, al manipular nuevos procedimientos endovideoscópicos o usar técnicas convencionales más simples ${ }^{11,12}$. Factores que dificultan el entrenamiento quirúrgico son la cambiante población hospitalaria internada como también el amplio rango de enfermedades con terapéuticas quirúrgicas cada vez más complejas ${ }^{13}$, con una presión creciente en la eficiencia o en la disminución de $\operatorname{costos}^{14}$.
La enseñanza de las maniobras quirúrgicas básicas que un residente de cirugía general debe conocer, manejar y adquirir como hábito de destreza y habilidad manual, puede implicar una dificultad no valorada desde el punto de vista ético en el clásico proceso de enseñanza-aprendizaje realizado con y sobre el paciente.

El objetivo del cuerpo docente, responsable de la formación quirúrgica, está centrado en que adquieran no sólo conocimientos teóricos sino también que desarrollen las destrezas necesarias. Un constante problema en la enseñanza de habilidades en los programas de residencias quirúrgicas es determinar su evaluación con procedimientos válidos y de valor reconocido ${ }^{15}$. Definir la naturaleza de la mejoría o adquisición de una habilidad es difícil de cuantificar, y por lo tanto es arduo de establecer cuál es el método de estimación apropiado.

Los programas de residencias quirúrgicas tradicionalmente usaron al quirófano como campo de enseñanza de destrezas; sin embargo, los residentes participan de un modelo didáctico basado no solo en el desarrollo de técnicas operatorias en pacientes, algunos con patología compleja, sino también en adquirir una responsabilidad progresiva e independiente ${ }^{16}$ que permita actuar sin perjudicar al enfermo.

Por lo tanto, el procedimiento de capacitación en servicio requiere del residente estar al lado del enfermo con un programa estandarizado posible de cumplir. Esta forma de enseñanza se basa en el procedimiento de los 4 pasos (mostrar, decir, hacer y confirmar), aplicable en forma progresiva y constante durante la Residencia ${ }^{17,18}$.

Es objetivo del presente trabajo preliminar evaluar características cualitativas de las destrezas y habilidades en 2 tipos de procedimientos técnicos básicos que pueden estar al alcance de un cirujano general: la toracotomía y la dermolipectomía. La finalidad última es definir la competencia en técnicas quirúrgicas de acceso.

La hipótesis a probar es demostrar, mediante un método observacional, la existencia de habilidades en los cirujanos formados durante un programa de residencia quirúrgica en el desarrollo de 2 técnicas no complejas.

\section{MATERIAL Y MÉTODO}

El presente estudio se realizó en forma prospectiva no randomizada a partir del 01 de junio de 2003 al 31 de mayo de 2004 en las Divisiones Cirugía 
Torácica y Cirugía Plástica del Hospital de Clínicas "José de San Martín".

El diseño es observacional, longitudinal, prospectivo: consistió en la observación de los pasos de una toracotomía axilar (técnica $\mathrm{N}^{\circ} 1$ ) en pacientes con patología torácica o de una dermolipectomía abdominal anterior con neo ombligo (técnica $\mathrm{N}^{\circ} 2$ ) en enfermos con patología morfológica de la pared abdominal anterior, realizadas por residentes de cirugía. Todas las operaciones eran programadas con antelación.

Se excluyeron las toracotomías con resección costal, las toracotomías mínimas o los enfermos con lesión tumoral de pared torácica. Fueron criterios de exclusión de dermolipectomía las minidermolipectomías, la técnica refuerzo de faja muscular (desinserción), los pacientes con obesidad mórbida o patología asociada de pared abdominal como hernia o eventración.

La muestra analizada fue de la práctica de las operaciones y no se investigaba quienes las habían efectuado (en más de una ocasión, un residente participó en varios procedimientos quirúrgicos): correspondieron a 7 residentes de cirugía general que cursaban el $3 \mathrm{er}$ o $4^{\circ}$ año durante su rotación por la División de Cirugía Torácica y 6 de cirugía plástica.

Los datos calificadores eran registrados mediante una lista de cotejos por los observadores/autores del presente estudio. Se utilizó una escala de medición tipo Likert modificada con sólo 3 ítems (elección del instrumental adecuado, ubicación espacial y número de intentos en realizar la maniobra) para calificar cada uno de los 4 pasos en la técnica quirúrgica realizada (incisión, disección de planos, hemostasia y síntesis de los planos músculo-aponeurótico, subcutáneo y epidérmico). La modificación de la escala de Likert consiste en valorar con sólo 4 puntos numéricos cada item evaluado (elección del instrumento, ubicación espacial, número de intentos) (en Metodología de la Investigación de Hernández Sampieri R, Fernandez Collado C y Baptista Lucio P. McGraw Hill Interamericana Editores, México, 2000, pag. 256). Se definió como:

- elección del instrumental adecuado a la maniobra de preferir un instrumento que permita realizar en forma apropiada aquello para lo cual está destinado hacer;

- ubicación espacial a la forma como se ubica el instrumento para realizar la maniobra correspondiente $\mathrm{y}$

- número de intentos en realizar la maniobra a la cantidad de veces que se probó hasta alcanzar desarrollar la maniobra conveniente.

Cada paso de la técnica quirúrgica practicada (toracotomía o dermolipectomía) se evaluó como:

- valor 3 : muy bueno

- valor 2: bueno

- valor 1: regular

- valor 0: deficiente.

Se consignaron, además, los valores totales de cada paso de la técnica en cada caso observado. No se consideró el tiempo de duración de cada maniobra quirúrgica.

Los evaluadores y autores del diseño, egresados de la Carrera Docente de la Facultad de Medicina de Buenos Aires, uno de ellos con una Maestría en Educación Universitaria (PFJ) y otro Profesor Adjunto de Cirugía, eran ajenos al equipo quirúrgico actuante y se encontraban en miradores donde no podían ser vistos por los residentes. Para evitar sesgos o maniobras que complicaran su programada actividad quirúrgica los participantes ignoraban que eran observados. Todos los datos que pudieran identificar a los participantes fueron reemplazados con un código numérico para asegurar la confidencialidad y evitar sesgos.

Fueron descartados los registros incompletos de las diferentes observaciones así como aquellos casos cuyas técnicas practicadas en una forma distinta a la programada.

\section{Análisis estadístico:}

Fue usado para este análisis el programa SPSS para Windows 10.0.

La consistencia interna (confiabilidad) del instrumento de evaluación (lista de cotejos de Likert modificada) y el correspondiente valor global de la escala en cada paso técnico-quirúrgico fue calculado con la prueba de alfa-Cronbach. Esta prueba sirve para calcular la propiedad de un instrumento de evaluación que permite la misma medición en ocasiones distintas. También se la llama confiabilidad de prueba-reprueba (Dawson-Saunders B, Trapp RG: Bioestadística médica. Manual Moderno, México, 1997, pg. 69). Un nivel de confiabilidad aceptable es de 0.8 o mayor.

Se utilizó la prueba de análisis de varianza (ANOVA) para demostrar las variaciones en el desempeño de cada paso en las 2 distintas técnicas quirúrgicas. Se usó la prueba de t de Student de muestras independientes para detectar cualquier diferencia en los resultados ("scores") de cada paso. Para todas las pruebas estadísticas mencionadas anteriormente, se consideró significativo un valor $\mathrm{p} £ 0.05$. 
Tabla 1: Calificación de destrezas en toracotomías

\begin{tabular}{lrrrr}
\hline \multicolumn{1}{c}{ ÍTEM } & $\begin{array}{r}\text { Elección } \\
\text { instrumento }\end{array}$ & $\begin{array}{r}\text { Ubicación } \\
\text { espacial }\end{array}$ & $N^{\circ}$ intentos & $\alpha C$ ronbach \\
\hline INCISIÓN & $2,391^{*}$ & $2,739^{*}$ & $2,652^{*}$ & 4,029 \\
DISECCIÓN & $2,478^{*}$ & $2,739^{*}$ & $2,739^{*}$ & 3,663 \\
HEMOSTASIA & $2,826^{*}$ & $2,695^{*}$ & $2,913^{*}$ & 0,932 \\
CIERRE &, $783^{*}$ & $2,739^{*}$ & $2,696^{*}$ & 2,75 \\
Promedio General & 2,6195 & 2,728 & 0,099007 \\
Desvío Standard & 0,1881 & 0,01905 & & \\
$*$ en promedio & & & &
\end{tabular}

\section{RESULTADOS}

En la tabla número 1 se detallan las características observadas para un total de 24 procedimientos (toracotomía axilar). Se puede apreciar que todos los pasos quirúrgicos, en todos los items investigados, fueron calificados en promedio por encima del valor 2 (bueno) y que este instrumento de evaluación era confiable en el análisis de la medición de las destrezas en los diferentes pasos según la prueba a de Cronbach. No había variaciones en el desarrollo de cada paso al evaluar las destrezas observadas y ésto era confirmado al probarlo con el "test" de ANOVA, con resultado de 0,7744 , "no significativo".

$\mathrm{Al}$ comparar los diferentes ítems analizados al practicar una toracotomía axilar mediante la prueba de t- Student se demostró que no había diferencias estadísticamente significativas en la comparación de destrezas como ser la elección del instrumento adecuado, la ubicación espacial en su uso y/o el número de intentos en realizar la maniobra quirúrgica hasta completar su acción determinada.

$\mathrm{Al}$ observar la dermolipectomía abdominal se apreció que todos los pasos quirúrgicos, en todos los ítems investigados, fueron en promedio calificados de manera similar a la otra técnica por encima del valor 2 (bueno) y que también era confiable este instrumento de evaluación (tabla número 2 ).
No hubo variaciones en los distintos pasos analizados, ratificado por la prueba de análisis de Varianza (ANOVA), con un valor de 0,00000727, "no significativo".

Mediante la prueba de t-Student se demostró que no hubo diferencias estadísticamente significativas en la comparación de destrezas.

\section{DISCUSIÓN}

El futuro practicante de la medicina científica debe estar en posición de aprender los principios esenciales de su práctica profesional ${ }^{19} \mathrm{y}$ hacer de su competencia, un hábito ${ }^{20,21}$. Sin embargo, en todo proceso de enseñanza-aprendizaje es necesario satisfacer un requisito insoslayable como es la evaluación permanente. Evaluar es, según el Diccionario de la Real Academia Española en su $23^{\text {a }}$ edición del 2003, señalar, estimar, calcular el valor de algo; en última instancia, valorar alguna cosa ó calificar el nivel alcanzado al ejecutar una destreza y/o habilidad manual.

La justificación de la Educación Continua ${ }^{22}$ debe buscarse en el cumplimiento de su objetivo primario que es mejorar la atención médica. Y eso sólo puede lograrse mediante un programa de enseñanza acorde con los contenidos teóricos y correlacionados con la práctica misma, que debe ser evalua-

\begin{tabular}{lrrrr}
\hline Tabla 2: Calificación de destrezas en dermolipectomías & & & \\
\hline PASO & $\begin{array}{r}\text { Elección } \\
\text { instrumento }\end{array}$ & $\begin{array}{r}\text { Ubicación } \\
\text { espacial }\end{array}$ & $\mathrm{N}^{\circ}$ intentos & $\alpha$ Cronbach \\
\hline INCISIÓN & $2,692^{*}$ & $2,4^{*}$ & $2,44^{*}$ & 5,984 \\
DISECCIÓN & $2,6^{*}$ & $2,44^{*}$ & $2,2^{*}$ & 272 \\
HEMOSTASIA & $2,6^{*}$ & $2,2^{*}$ & $2,12^{*}$ & 6,016 \\
CIERRE & $2,36^{*}$ & $2,44^{*}$ & $2,32^{*}$ & 15,45 \\
Promedio General & 2,563 & 2,37 & 2,27 & \\
Desvío Standard & 0,1231 & 0,09949 & 0,1212 & \\
* en promedio & & & &
\end{tabular}


do en forma sistemática y permanente ${ }^{23}$. Las cualidades deseables de encontrar en los cirujanos son ${ }^{24}$ la destreza, su capacidad de decisión, poseer buena pericia técnica, habilidad de trabajar en equipo y de resolver problemas. Para asegurar estas características, es imprescindible definir los métodos que aseguren su aprendizaje y especificar su forma de evaluación. Se debe tener en cuenta, además, el costo del entrenamiento quirúrgico de residentes en los quirófanos, con los aspectos éticos no bien justipreciados en esta forma de atención sanitaria. Ese costo es demasiado alto aún en Estados Unidos de Norteamérica (alrededor de u\$s 53 millones anuales) y en ocasiones no evitan los errores en la atención médica ${ }^{25,26,27}$.

La formación del médico residente de cirugía debe estar contenida en el marco de la educación médica como actividad integrada de un sistema.

Pero ¿cómo se reconoce la existencia de destrezas y habilidades enseñadas durante el programa de residencia, que efectivamente han sido aprendidas, si se desconoce el método para valorarlas? ¿O es acaso, simplemente, un ejercicio de conocimientos teóricos más que prácticos los analizados durante la evaluación?

Algunos estudios efectúan la medición de destrezas mediante evaluación puramente teórica ${ }^{28,29}$. Por otra parte, las opiniones de los encuestados por el Departamento Universitario de Cirugía de Edimburgo, utilizando una compleja técnica Delphi de encuestas acerca de las destrezas necesarias para un adiestramiento quirúrgico básico ${ }^{30}$, demostraron que luego de 6 meses de instrucción elemental se identificaron las cualidades adquiridas y esas eran: comunicación con pacientes y familiares, aplicación del conocimiento teórico, destrezas clínicas no especificadas, trabajo en equipo y habilidades técnicas sencillas. Llama la atención que las habilidades específicamente técnicas individuales no fueron las señaladas como más importantes, sino aquellas que demandaban trabajar en equipo.

El proceso de enseñanza - aprendizaje va más allá de una simple transmisión - adquisición de conocimientos. Implica el desarrollo de las tres áreas de la conducta: cognoscitiva, socioafectiva y psicomotriz ${ }^{31}$. Si se recuerda aquel aforismo atribuido a Confucio que"si escuchamos, podemos olvidar; si vemos, podemos recordar pero si hacemos, seguro que comprenderemos" ${ }^{18}$, se jerarquizará el real valor de la evaluación de destrezas adquiridas.

Por lo expresado, es fundamental para cualquier método de evaluación una determinación precisa y clara de las destrezas y habilidades necesarias para conseguir la competencia quirúrgica buscada .

En la investigación aquí presentada no se realizó la predicción posible de la "performance" en el futuro ${ }^{32} \mathrm{ni}$ su correlación con los conocimientos teóricos como sí lo sostuvieron otros autores ${ }^{7}$, porque no era ése el objetivo del estudio.

Es interesante enumerar diversas mediciones efectuadas por otros autores como maneras de evaluar destrezas, y considerando otros factores: efecto del cansancio o la falta de sueño de los residentes participantes ${ }^{33}$, participación previa en cursos de entrenamiento manual ${ }^{34,35}$ con cadáveres ${ }^{36,37}$, ensayos de "banco de laboratorio" ${ }^{38}$ como puede ser una cateterización venosa central ${ }^{39}$, modelos de simulación humanos ${ }^{40,41}$ en algunos casos portátiles (los llamados "muñecos" ${ }^{42}$ ), con fotografías o diapositivas de endoscopias ${ }^{43} \mathrm{y}$ analizados por programas de computación en algunos centros de entrenamiento ${ }^{44}$.

De igual forma puede implementarse la observación de videos ${ }^{45,46}$ o la presencia de docentes cirujanos que encarnan modelos a imitar por su reconocida y demostrada habilidad técnico-quirúrgica ${ }^{47}$. Asimismo se descartó la posibilidad de examinar las habilidades manuales con pruebas clínicas estructuradas objetivas como se empleó en la Universidad de Kentucky ${ }^{48}$ o en el Departamento de Cirugía de la Universidad de California ${ }^{49}$ o el uso de cuestionarios basados en las observaciones del quirófano ${ }^{50}$. Se excluyó el uso de modernos métodos de material didáctico provisto en CD-ROM con los conceptos quirúrgicos básicos de habilidades ${ }^{51}$.

Una manera de vigorizar el aprendizaje mediante la práctica, es crear potenciales escenarios de adquisición de habilidades, con consideración de las relaciones visual-espacial ${ }^{52,54}$, como pueden ser las simulaciones virtuales aún puestas de manifiesto desde el pregrado como bien lo expuso Ricardo Torres en la Universidad de Corrientes en Argentina ${ }^{53}$ o ensayar con casos inconfundibles como modelos de adiestramiento práctico.

Ante la aparición de nuevos procedimientos quirúrgicos ${ }^{55}$, siempre se debe enseñar y aprender con seguridad y eficiencia ya que al operar pacientes se considera que la curva de aprendizaje ha llegado a su nivel de competencia.

Por otro lado, la confusión semántica entre desempeño (performance), pericia y habilidad contribuye a no discernir la forma de calificar la actividad práctica individual. Así se define al desempeño como la eficiencia global con la cual una actividad se ejecuta; la pericia es un componente de un desem- 
peño basado tanto en la experiencia como en la capacidad de hacerla y la habilidad es aquella que se obtiene por la práctica constante de un movimiento, maniobra o gesto, en este caso relacionado con la cirugía. Así, para pasar un punto de sutura un cirujano formado no desdobla sus movimientos sino que al haber adquirido un gesto quirúrgico, efectúa el movimiento en forma automática y lo aplica a los fines determinados previamente, según la técnica que debe realizar ${ }^{56}$.

La presente investigación de carácter exploratorio es diferente a lo anteriormente visto por otros autores. Se intentó únicamente demostrar la presencia de habilidades en médicos residentes de 3er ó $4^{\circ}$ año en un servicio de Cirugía General de un hospital universitario en técnicas quirúrgicas no complejas, basándose exclusivamente en la observación de los distintos movimientos que realizaban durante el desarrollo de las mismas. Con anterioridad uno de los autores (PFJ) ${ }^{23}$ había observado que un entrenamiento programado en microcirugía experimental, antes de realizar ciertas operaciones en pacientes, era un medio eficaz para lograr un desarrollo del área psicomotriz de la conducta y obtener una instrucción segura y rápida de destrezas quirúrgicas. Actualmente, sólo se quiso señalar la presencia de esas habilidades en los residentes de años superiores en una residencia acreditada. Si bien se hizo con residentes de 2 distintas especialidades quirúrgicas, una de ellas (cirugía plástica) era de un segundo nivel superior, porque sus integrantes tenían como condición previa el haber completado una residencia quirúrgica de 3 años. Sin embargo, no se quiso señalar si había un grupo mejor que otro, por ser de diferentes niveles y no poder afirmarse cuál era el llamado grupo control.

La elección del instrumental adecuado, su manejo y ubicación espacial en los planos tegumentarios y el número de intentos en realizar una maniobra determinada son, en definitiva, las actividades surgidas como una serie de componentes elementales y necesarios, dispuestos en un orden preciso y lógico. La investigación de estas acciones seleccionadas surgen de estudios previos ${ }^{57,58}$ que, como se mencionó, son raramente evaluadas. El esquema de $K$ opta $^{59}$ para el desarrollo de una habilidad técnica señala 3 grandes momentos: percepción, integración y automatización, que son secuencialmente seguidos durante el aprendizaje de un gesto, en este caso quirúrgico y eso es lo que se quiso comprobar mediante la simple observación de las maniobras efectuadas de los residentes. Se eligieron manio- bras quirúrgicas perfectamente definidas como la incisión, disección de planos tegumentarios, hemostasia y síntesis, por ser movimientos comunes a las 2 diferentes técnicas quirúrgicas aquí evaluadas.

El criterio de medir el desempeño con una escala de Likert modificada en los distintos componentes de las 2 técnicas quirúrgicas, fue debido a que esta metodología de lista de cotejos permite una fácil y rápida evaluación cuantitativa de los mismos, como ya fue corroborado por otros autores 6 . El uso de la prueba a-de Cronbach, permitió asegurar la validez y confiabilidad de las mediciones con el instrumento elegido (la escala de Likert modificada) en la totalidad de las observaciones realizadas $(n=24$ toracotomías y $\mathrm{n}=25$ dermolipectomías abdominales), a pesar de ser 2 investigadores diferentes.

No hubo diferencias estadísticamente significativas entre los distintos ítems y pasos quirúrgicos. Y esto es así porque de alguna manera, los participantes observados se encontraban en los años superiores de sus respectivos programas de residencia y mostraban una real adquisición de habilidades, a pesar que desde 1983 Spencer $^{60}$ señalaba que las habilidades requeridas para desempeñar un procedimiento quirúrgico representaban sólo un $25 \%$ y lo más importante era fundamentalmente la toma de decisiones.

La realización apropiada de las incisiones operatorias, por ejemplo, su real ubicación espacial al emplear el instrumental elegido o el desempeño ordenado sin exceso del número de intentos para completar un gesto quirúrgico se destacaron como buenos, coincidentes con otros autores que encuestaban a cirujanos consultores que destacaban estas mismas cualidades con deducciones similares ${ }^{24,61}$.

Los resultados aquí presentados, si bien pueden considerarse de un valor relativo dentro de un programa general de una residencia quirúrgica, nunca antes habían sido puestos en evidencia en nuestro país con una estimación cuantitativa simple y de fácil recolección. Esta metodología permite calcular repetidas veces el desempeño de los residentes y definir su seguimiento durante todo su período de entrenamiento.

La clara intencionalidad de los autores de no ser observados por quienes estaban siendo evaluados en sus habilidades era para evitar la aparición de situaciones sesgadas conocidas como "efecto Hawthorne" ${ }^{18}$, donde quien se sabe "vigilado" se comporta de una manera muy diferente a la habitual. De la misma manera, no medir la duración de cada gesto quirúrgico en cada una de las 49 opera- 
ciones observadas (24 de cirugía torácica y 25 de cirugía plástica) era para obviar un sesgo similar de competición, sólo presente en el pensamiento del autor/evaluador.

La real trascendencia de los resultados aquí obtenidos podrá facilitar la estimación del desempeño de un residente quirúrgico en formación, con responsabilidad progresiva e independiente en la atención de pacientes.

Las limitaciones del análisis exploratorio presentado pueden deberse a las dificultades en valorar actividades manuales en los quirófanos, muchas veces por carecer de planeamiento educativo adecuado.

Se trata de superar la mera evaluación subjetiva de los residentes, que como técnica es de validez imprecisa y de pobre confiabilidad, como ya fue observada en otras especialidades quirúrgicas ${ }^{62}$. La posibilidad de cambios en el currículo a partir de hallazgos como los mencionados en este análisis, permitirán evaluar de manera más objetiva y sin sesgos las habilidades quirúrgicas.

La presunción de que cursos de "fin de semana" son claros sustitutos de la experiencia práctica continua y avalada por criterios instruccionales creíbles y posibles de cumplir durante un tiempo mayor a un par de días, hacen vulnerable y cuestionable nuestra competencia como cirujanos. Sólo la observación directa de las habilidades permite su evaluación.

La evaluación de las habilidades quirúrgicas adquiridas es un requisito primordial en el análisis de nuestra competencia.

El sencillo método de evaluación aquí propuesto (observación inadvertida por el residente en entrenamiento y que es evaluado) puede facilitar este proceso educativo y permitir la real certificación de habilidades adquiridas. La necesidad de un mayor número de observaciones precisará la verdadera naturaleza e importancia de este sencillo método evaluador.

\section{BIBLIOGRAFÍA}

1. Weed LL. New connections between medical knowledge and patient care. BMJ, 1997;315:231-5.

2. Burt CG, Chambers E et al. The evaluation of a new method of operative competence assessment for surgical trainees. Bull Roy Coll Surg Engl, 2003;85(5):152-5.

3. Scheinfeld AR de: La teoría de Howard Gardner y la educación universitaria. Rev Científica UCES, 1999;III(1):175-178.

4. Iribarren C, Arribalzaga EB y Curutchet HP. Error médico en cirugía. Rev Argent Cirug, 2003;85(3-4):124-134.

5. Adams JA. Historical review and appraisal of research on the learning, retention and transfer of human skills. Psychol Bull, 1987;101:41-74.

6. Schueneman AL, Picklemn J, Hesslein R and Freeark RJ:
Neuropsychologic predictors of operative skill among general surgery residents. Surgery, 1984;96(2):288-295.

7. Willoughby TL, Gammon LC, Jonas HS. Correlates of clinical performance during medical school. J Med Educ 1979;54:45360 .

8. Alenta HP: Formación continuada, desarrollo profesional y recertificación de los médicos. Educ Méd, 2003;6(4):123-124.

9. Schwind CJ, Boehler ML et al: variables influencing medical student learning in the operating room. Am J Surg, 2004;187:198-200.

10. Hamdorf JM and Hall JC: Acquiring surgical skills. Br J Surg, 2000;87:28-37.

11. Anastakis DJ, Regehr G et al: Assessment skills transfer from the bench training model human model. Am J Surg, 1999;177:167-170.

12. Thornton M, Donlon M, Beard JD. The operative skills of higher surgical trainees: measuring competence achieved rather than experience undertaken.Bull Roy Coll Surg Engl, 2003;85(6):190-218.

13. Haluck RS and Krummel TM: Computers and virtual reality for surgical education in the 21st Century.Arch Surg, 2000;135:786-792.

14. Bridges M, Diamond DL. The financial impact of teaching surgical residents in operating room. Am J Surg, 1999;177:28-32.

15. Rogers DA, Elstein AS and Bordage G: Improving continuing medical education for surgical techniques:applying the lessons learned in the first decade of minimal access surgery. Ann Surg, 2001;233(2):159-166.

16. Santas AA: Función de la residencia en la formación del médico. Rev Argent Cirug, 1966;10(1):43-45.

17. Anastakis DJ, Hamstra SJ, Matsumoto ED: Visul-spatial abilities in surgical training. Am J Surg, 2000;179:469-471.

18. Arribalzaga EB: Curriculum en la especialidad cirugía torácica. La residencia. En: Cherjovsky R, Arribalzaga EB y Gomez PA. Currículo en la especialidad cirugía torácica. Edit Nobuko/UAI, 2003, Buenos Aires, p. 53-105.

19. Miettinen OS: The modern scientific physician: 8. educational preparation. CMAJ, 2001;165(11)1501-1503.

20. Leach DC. Competence is a habit. JAMA, 2002;287:243-4.

21. Lypson ML, Frohma JG, Gruppen LD and Woolliscroft JO: Assessing residents' competencies at baseline: identifying the gaps. Acad Med, 2004;79:564-570.

22. Reznick RK. Let's not forget that CME has an "E". Foc Surg Ed, 1999;17:1-2.

23. Jacovella PF: Las maniobras quirúrgicas en cirugía general. Análisis del desarrollo de habilidades. Rev Argent Cirug, 1991;60(1-2): 53-62.

24. Baldwin PJ, Paisley AM and Paterson Brown S: Consultant surgeons' opinion of the skills required of basic surgical trainees. Br J Surg,1999;86:1078-1082.

25. Kempainen RR, Migeon MB and Wolf FM: Understanding our mistakes: a primer on errors in clinical reasoning. Medical Teacher, 2003;25(2):177-181.

26. Rogers DA, Regehr G, MacDonald J: A role for error training in surgical technicl skill instruction and evaluation. Am J Surg, 2002;183:242-245.

27. Sarker SK: Courses, cadavers and counsellors:reducing errors in the operating theatre. BMJ , 2003;327:s109.

28. Boehler ML, Rogers DA et al. A senior elective designed to 
prepare medical students for surgical residency. Am J Surg, 2004;187:695-7.

29. Bull DA, Stringham JC, Karwande SV and Neumayer LA: Effect of a resident self-study and presentation program on performance on the thoracic surgery in-training examination. Am J Surg, 2001;181:142-144.

30. Driscoll PJ, Paisley AM and Paterson-Brown S: Trainees' opinions of the skills required of Basic surgical trainees. Am J Surg, 2003;186:77-80.

31. Epstein CD, Hovancsek MT et al. Lights !Camera !Action !: video projects in the classroom.J Nurs Educ, 2003;42(12:558 561.

32. Ozuah PO: Predicting residents' performance: a prospective study. BMC Medical Education, 2002;2(7):1-4. Available from URL http://www.biomedcentral.com/1472- 6920/2/7.

33. Grewal H. Temple university; sleep deficits don't hinder learning in surgical residents. Biotech Week, Atlanta, Jul 14, 2004, pg 522 .

34. Datta V, Mandalia M et al: Relationship between skill and model outcome in the laboratory-based model. Surgery, 2002;131:318-23.

35. Ratnapalan S and Hilliard RI: Needs assessment in postgraduate medical education: a review. Med Educ Online 2002;7 (8):1-8. Available from URL http:// www.med-ed-online.org.

36. Anastakis DJ, Wanzel KR et al: Evaluating the effectiveness of a 2-year curriculum in a surgical skills center. Am J Surg, 2003; 185:378-385.

37. Jones LS, Paulman LE, Thadani R and Terracio L : Medical students dissection of cadavers improves performance on practical exams but not on the NBME anatomy subject exam. Med Educ Online, 2001;6(2):1-8. Available from URL http://www.med-ed-online.org.

38. Schwind CJ, Boehler ML et al: Development of physical examination skills in third-year surgical clerkship. Am J Surg, 2001;181:338-340.

39. Velmahos GC, Toutouzas KG et al. Cognitive task analysis for teaching technical skills in an inanimate surgical skills laboratory. Am J Surg, 2004;187:114-9.

40. Nackman GB, Bermann M and Hammond J. Effective use of human simulators in surgical education. J Surg Research, 2003;115:214-8.

41 Paisley AM, Baldwin PJ, Paterson-Brown S. Validity of surgical simulation for the assessment of operative skills. Br J Surg, 2001;88:1525-1532.

42. Schell SR and Flynn TC. Web-based minimally invasive surgery training: competency assessment in PGY 1-2 surgical residents. Curr Surg, 2004;61:120-4.

43. Sammarco MJ, Youngblood JP: A resident teaching program in operative endoscopy. Obstet Gynecol 1993;81:463-6.
44. Wanzel KR, Matsumoto ED et al: Teaching technical skills: training on a simple, inexpensive and portable model. Plast Reconstr Surg, 2002;109:258-264.

45. Heppell J, Beauchamp G and Chollet A: Ten-year experience with a basic tachnical skills and perioperative management workshop for first-year residents. Can J Surg, 1995;38:27-32.

46. Kneebone RL. Twelve tips on teaching basic surgical skills using simulation and multimedia. Medical Teacher, 1999;21(6):571-6.

47. Maker VK, Curtis KD and Donnelly MB. Are you a surgical role model?Curr Surg, 2004;61:111-5.

48. Warf BC, Donnelly MB, Schwartz RW and Sloan DA: Interpreting the judgement of surgical faculty regarding resident competence. J Surg Research, 1999;86:29-35.

49. Lazar HI, DeLand EC and Tompkins RK: Clinical performance versus in-training examinations as measures of surgical competence. Surgery, 1980;87(4):357-362.

50. Evans A. Helping GP trainers to improve their random case analysis teaching skills: development of a programme. Educ Prim Care, 2004;15(3):336-343.

51. Sherris DA and Kern EB. Basic surgical skills. 1999, Mayo Clinic Scientific Press, Rochester.

52. Smith V. A learner-centred model of training practice inspection: in-depth interview study of GP registrars'perceptions of the learning climate of their training year. Educ Prim Care, 2004;15(3):361-369.

53. Torres RA, Orban RD y col: Enseñanza de técnicas quirúrgicas básicas en simuladores biológicos. Experiencia pedagógica en el pregrado. Educ Méd, 2003;6(4):149-152.

54. Wanzel KR, Hamstra SJ et al: Effect of visual-spatial ability on learning of spatially-Complex Surgical skills. Lancet, 2002;359(9302):230-1.

55. Gates EA. New surgical procedures: can our patients benefit while we learn? Am J Obstet Gynecol, 1997;176:1293-9.

56. Edgerton MT. The art of surgical technique. Williams and Wilkins, Baltimore, 1988, pg. 3-29.

57. Hall JC: Imagery practice and the development of surgical skills. Am J Surg, 2002;184:465-470.

58. Reznick RK: Teaching and testing technical skills. Am J Surg, 1993;165:358-361.

59. Kopta JA: An approach to the evaluation of operative skills. Surgery, 1971;70(2):297-303.

60. Spencer FC. Observations on the teaching of operative technique. Bull Am Coll Surg, 1983;68:3-6.

61. Satava RM, Gallagher G and Pellegrini CA. Surgical competence and surgical proficiency: definitions, taxonomy and metrics. J Am Coll Surg, 2003;196:933.

62. Mandel LP, Lentz GM and Goff BA: Teaching and evaluating surgical skills. Obstet

Gynecol, 2000;95:783-5. 